\title{
Les résidus post-mictionnels
}

\section{Post void residual urine volume}

\section{G. Amarenco}

Reçu le 8 mars 2010 ; accepté le 9 mars 2010

(C) Springer-Verlag France 2010

\section{"Le résidu n'a pas d'importance s'il n'est pas compliqué » A. Leriche.}

Le résidu témoigne d'une rétention chronique si tant est qu'il soit... permanent !

En effet, combien de patients arrivent en consultation, effrayés eux-mêmes ou par leur généraliste (voire leur radiologue...), par une échographie vésicale post-mictionnelle effectuée... de très nombreuses minutes après une miction, souvent effectuée difficilement chez un patient qui s'est trop longtemps retenu et, dans la plupart des cas, après une très large hyperhydratation. C'est dans ces cas que le diagnostic de résidu est porté en excès, diagnostic aisément redressé par un sondage transurétral immédiatement post-mictionnel ou par quelques échographies post-mictionnelles effectuées dans des conditions de miction plus propices à une physiologie normale (miction relâchée, sur besoin, en réplétion vésicale normale, sans surdistension et dans des conditions de confort et d'isolement acceptables).

Si une rétention chronique est néanmoins confirmée, un certain nombre de problèmes se posent et, en premier lieu, l'existence de complications éventuelles qui peuvent lui en être attribuées : pollakiurie réactionnelle (et notamment nycturie), fuites par regorgement pouvant en imposer pour une « banale » incontinence urinaire (notamment chez la personne âgée), infections urinaires récurrentes. C'est dans ces cas et ces cas seulement qu'un traitement s'impose. Ce sont bien les symptômes induits qui dictent le traitement et non pas la rétention elle-même. Ce traitement repose le plus souvent sur un traitement étiologique spécifique (levée d'une obstruction, suppression d'épines irritatives, vérification des médications potentiellement rétentiogènes). Mais parfois, et c'est souvent le cas des vessies neurogènes, le traitement symptomatique est indispensable et s'appuie sur la réalisation d'autosondages (voire hétérosondages transitoires chez la personne très âgée), les traitements médicamenteux (alphabloquant, injection intrasphinctérienne de toxine botulique, parasympathomimétiques direct ou indirect) étant le plus souvent inefficaces, et la neuromodulation des racines sacrées parfois lourde à mettre en œuvre pour une telle indication.

Mais le résidu n'est pas la dysurie... Ce résidu pourrait être en effet « respecté » en l'absence des complications suscitées, mais la seule présence d'une dysurie sans rétention, par les poussées abdominales qu'elle signifie, justifie parfois un traitement pour éviter reflux et risque de prolapsus, notamment chez la femme. Le problème est alors la quantification de ces poussées abdominales réalisée au mieux par des études pression-débit spécifiques, voire par une simple débitmétrie effectuée avec un couplage à une pression rectale (ou vaginale) ou à une électromyographie abdominale de surface.

Enfin, le résidu ne peut s'appréhender seul. Il fait partie d'un équilibre général. Et s'il est associé à d'autres conditions pathologiques (hyperactivité détrusorienne, obstruction fonctionnelle ou organique), même en l'absence de complications directes, un traitement va s'imposer, traitement qui d'ailleurs le majore souvent (anticholinergiques pour une hyperactivité vésicale) quand il ne le créait pas (toxine botulique). Dépister et affirmer le résidu, l'évaluer, en rechercher les éventuelles complications, faire la part des autres conditions physiopathologiques associées, telles que l'hyperactivité détrusorienne, sont ainsi tous les éléments à considérer avant de décider de son traitement qui ne doit pas être systématique.

\section{Conflit d'intérêt : aucun.}

G. Amarenco $(\square)$

Hôpital Rothschild

Service d'exploration périnéale et de rééducation neurologique

33 boulevard Picpus

75012 Paris

e-mail : gerard.amarenco@tnn.aphp.fr 